\title{
Sliding Mode Strategy for PEM Fuel Cells Stacks Breathing Control Using a Super-Twisting Algorithm
}

\author{
Cristian Kunusch, Member, IEEE, Paul F. Puleston, Miguel A. Mayosky, Senior Member, IEEE, and Jordi Riera
}

\begin{abstract}
A second-order sliding mode strategy to control the breathing subsystem of a polymer electrolyte membrane fuel cell stack for transportation applications is presented. The controller is developed from a design model of the plant derived from open literature, and well suited for the design of second-order sliding mode strategies. Stability issues are solved using a super twisting algorithm. The resulting approach exhibits good dynamic characteristics, being robust to uncertainties and disturbances. Simulations results are provided, showing the feasibility of the approach.
\end{abstract}

Index Terms-Energy conversion, fuel cells (FCs), hydrogen, nonlinear systems, sliding mode (SM) control.

\section{INTRODUCTION}

$\mathbf{F}$ UEL CELLS (FCs) are devices that convert hydrogen chemical energy into electric energy and can be used in a wide variety of applications, from mobile and stationary power systems to portable appliances. The FC principle of operation was discovered as early as 1839 , but only during the last two decades the research activity in this field increased remarkably, improving FC flexibility and reliability [1]. One of the most influential factors that soared FC development is the severe environmental impact of fossil fuel combustion. Considering that hydrogen production from water electrolysis can be performed using renewable energy (solar, wind, geothermal, etc.), polymer electrolyte membrane (PEM) fuel cells emerge as one of the most clean and promising alternatives to reduce fossil fuel dependency [2].

Improvements in this field require interdisciplinary work and development of new technologies in many areas. One of the most important issues is related to the development of robust control strategies dealing with disturbances and model uncertainties in a systematic way. For instance, effective control algorithms for the fuel-oxydant coordination problem inside the cells during variable load tracking, can avoid transient power deterioration and irreversible damages in cell membranes.

However, from the control point of view fuel cell stacks represent a major challenge because their associated subsystems present conflicting control objectives and complex dynamics [3]. For example, a ninth-order nonlinear model is used to describe a generation system based on a hydrogen-air fed stack. In such model the states interaction is usually modelled through

Manuscript received May 21, 2007; revised November 05, 2007. Manuscript received in final form February 21, 2008. First published May 14, 2008; current version published December 24, 2008. Recommended by Associate Editor M. L. Corradini. This work was supported by UNLP, CONICET, CICPBA, and CECyT, UPC, and AECI.

C. Kunusch, P. F. Puleston, and M. A. Mayosky are with the Universidad Nacional de La Plata (UNLP), La Plata 1900, Argentina (e-mail: ckunusch@ing. unlp.edu.ar; puleston@ing.unlp.edu.ar; mayosky@ing.unlp.edu.ar).

J. Riera is with the Universidat Politècnica de Catalunya (UPC), Institut de Robòtica i Informàtica Industrial (IRI), Parc Tecnològic de Barcelona, Carrer Llorens i Artigas 08028, Barcelona (e-mail: riera@iri.upc.edu).

Digital Object Identifier 10.1109/TCST.2008.922504 several lookup tables and decision blocks. Many internal variables are inaccessible for measuring and cannot be used in control algorithms. Besides, there are measurable and unmeasurable disturbances that can affect the system operation, as well as uncertainties in model parameters.

Therefore, in order to achieve an efficient controller for the FC system, it is required to rely on an adequate technique capable to cope with such challenges. In particular, the algorithms that belong to the family of high order sliding mode (SM) controllers represent an interesting option. Utilizing a reduced number of measured variables, they can solve the nonlinear robust stability of the system, converge to the reference in finite time and avoid chattering effects [4]. Then, the novelty and main contribution of this paper consists in using second-order sliding modes for the control of PEM fuel cells breathing. In this framework, a robust controller is developed to regulate the oxygenation of fuel cell cathodes through a turbo compressor. The controlled output has relative degree one with respect to the control input, so a super twisting algorithm is used to stabilize the system avoiding chattering as stated in [5]-[7]. The super twisting controller maintains the distinctive robust features of the SM techniques, while providing a control signal smoother than the one obtained via standard first-order SM. Such smoother control action represents an advantage not only from the chattering viewpoint, but also for the implementation of the electric drive of the compressor. In addition, due to the fact that the control input (i.e., the compressor voltage) has a direct influence on the electric output variables of the overall FC system, the absence of discontinuous components in the super twisting control action results into another beneficial feature regarding the output electric quality of this particular application.

Summarizing, the resulting controller robustly solves one of the most important problems related to PEM fuel cell systems fed by high pressure hydrogen and air turbo compressors. It guarantees maximization of power generation efficiency, while preventing oxygen starvation inside the stack, even in the presence of uncertainties, disturbances, and variable demand.

\section{SYSTEM DESCRIPTION AND CONTROL OVERVIEW}

\section{A. System Model}

There are several different approaches and models to describe the dynamic behavior of a fuel cell stack connected to a compressor, but only a few are suitable for control purposes. Among them, one of the most complete and accurate models available in the open literature was developed by J. Pukrushpan et al. in the Mechanical Department of the Michigan University. This model provides a detailed description of the dynamics of a 75-kW-high pressure FC stack fed by a 14-kW air turbo compressor (see Fig. 1), where the physical parameters are calibrated based on data reported in the literature. The system is sized to represent 
Fuel Cell Based Generation System

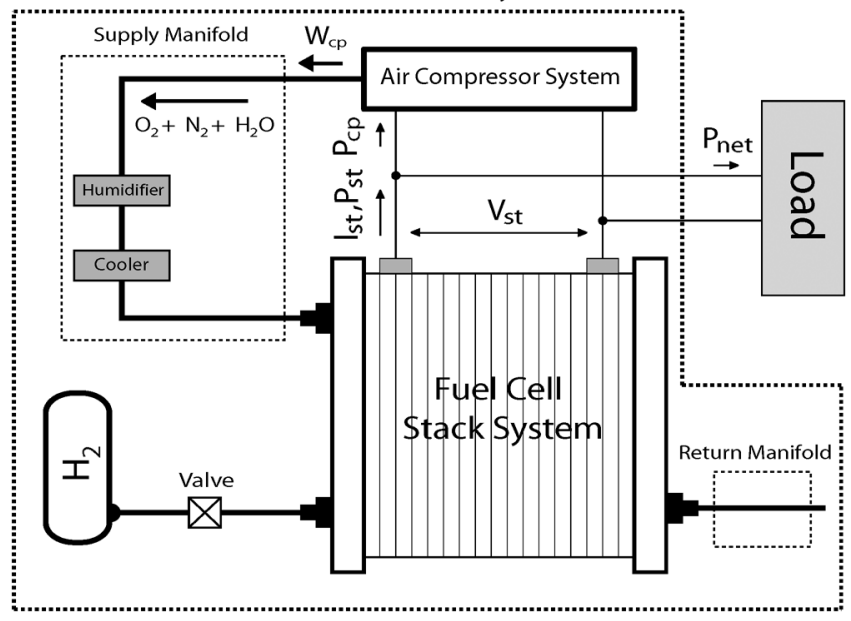

Fig. 1. Schematic diagram of a FCGS.

the high pressure FC stack used in the Ford P2000 fuel cell electric vehicle [3]. The dynamics of the fuel cell based generation system (FCGS) are divided into four different interconnected subsystems: the air flow (breathing), the hydrogen flow, the humidity, and the stack temperature subsystem, respectively. The system is described by a ninth-order nonlinear model. In [3], it is assumed that the stack temperature is well regulated and the input reactant flows can be humidified in a consistent and rapid way. In addition, it is considered that compressed hydrogen is available, therefore it focuses on the air management.

The breathing subsystem control input is the compressor DC motor voltage $\left(V_{\mathrm{cm}}\right)$. The differential equations of the model are obtained by considering: 1 ) the mass conservation equations for oxygen, nitrogen and water mass inside the cathode; 2) the rate of change of mass and pressure of the cathode supply manifold; 3 ) the rotational dynamics of the compressor motor; 4) the pressure of the cathode return manifold; and 5) the rate of change of hydrogen and water mass in the anode. Among the measurable variables of the system are the stack voltage $\left(V_{\mathrm{st}}\right)$, the oxygen mass flow delivered by the compressor $\left(W_{\mathrm{cp}}\right)$, the total current drained from the stack $\left(I_{\mathrm{st}}\right)$, and the net power generated by the system $\left(P_{\text {net }}\right)$. Note that the air compression system is connected in parallel to the load, acting as a parasitic load to the fuel cell stack. Therefore, the output net power of the FCGS is given by the difference between the fuel cell stack power and the power required by the compressor $\left(P_{\text {net }}=P_{\mathrm{st}}-P_{\mathrm{cp}}\right.$, see Fig. 1). It can be seen that the overall efficiency of the FCGS also depends on the compressor power demand.

Due to space limitations a detailed description of this model is not included in this brief paper, but it can be found in [3] and [8].

\section{B. Sliding Mode Control}

During the last decades, the control of systems under heavy uncertainty conditions received significant attention from the scientific community. Among the existing control options, the variable structure control (VSC) or sliding mode control proves to be a useful choice to work with nonlinear uncertain systems [9]. The control algorithms designed via SM control techniques can be relatively simple to design and implement, providing robustness to external disturbances or parameters changes [10], [11]. Reliability of VSC applied to electrical and mechanical systems has been largely reported in the literature [12]. The VSC is characterized by a control structure that switches as the system crosses certain manifold in the state space. The switching function (or sliding variable) $s$ is a function of the states, and the switching manifold (or sliding surface) is defined for $s=0$. The latter represents a static relationship between the state variables, hence reduced order dynamics are attained. These reduced order dynamics may provide attractive features, such as high performance, including insensitivity to parameters variations and disturbances that verify the matching condition [11]. Then, the basic idea of SM control is to design an adequate switching variable $s$, according with the desired control objectives, and a VSC action capable to drive and confine the system motion on the sliding surface.

However, a great deal of the controller success to fulfil the objectives strongly depends on the ability of the control to reduce the high frequency oscillations (chattering) on the system outputs. A useful alternative to cope with this undesirable effect is to steer to zero a certain number of time derivates of the sliding variable, smoothing the control action and counteracting the chattering effect in the outputs [7], [13]. This strategy is referred as higher order SM (HOSM), dynamic SM, or terminal SM. In particular, the HOSM controllers that steer to zero the sliding variable and its first derivate (both continuous), conform the family of second-order SM (SOSM) controllers. As it was previously mentioned, among the advantages of this solution it can be highlighted the capability of system robust stabilization, finite time convergence to the sliding manifold, chattering reduction, and good performance even in the presence of model uncertainties and disturbances [6], [14], [15]. Another SOSM feature of relevance for FC applications is the control design based on nonlinear models. Given the highly nonlinear nature of the fuel cell stack, this feature guarantees robust operation and performance in a more extended range than those control methods based on model linearization at different operation points. In addition, the SOSM algorithms are relatively simple, have a reduced set of controller parameters and rely on the information of few measurable variables, rather than on the knowledge of the full state vector, as it is required by some other control approaches.

\section{Control System Design}

\section{A. Design Model}

The first stage to perform a nonlinear control design of the system based on SOSM, is to reformulate the model description according with the requirements of the SOSM control design procedure, replacing the tabulated data and piece-wise continuous expressions by smooth functions. In the first place, Pukrushpan's model comprises a double input lookup table and switched piece-wise functions, all of them continuos but not differentiable (see the detailed information in [8]). Then, in the proposed design model they have been replaced with smooth functions, utilizing adequate polynomial approximations. Second, an order reduction based on control considerations 
of the physics of the system has been done (see the whole rebuilding work in [2]). Any minor degree of uncertainty generated by the aforementioned modeling procedure would be added to the inherent uncertainty of the original model. So, for the design of the proposed controller, such lumped uncertainties together with external disturbances and set point changes will be considered.

As a result of the rebuilding work performed over Pukrushpan's model, the following design model is proposed for SOSM design (see equations in Appendix II):

$$
\begin{aligned}
& \dot{x}=f(x(t))+g(x(t), u(t)) \\
& x \in \mathcal{R}^{6} ; u \in \mathcal{R} ; f: \mathcal{R}^{6} \rightarrow \mathcal{R}^{6} ; g: \mathcal{R}^{6} \rightarrow \mathcal{R}^{6}
\end{aligned}
$$

where the coordinates of the states vector can be summarized as follows:

\section{$x_{1}=\omega_{\mathrm{cp}}$ : angular speed of the compressor motor that} feeds the stack cathode through the supply manifold.

$x_{2}=p_{\mathrm{sm}}$ : total pressure inside the supply manifold, consisting of the sum of the partial pressures of the gases that constitute the air (oxygen, nitrogen and water vapor).

$x_{3}=m_{s m}$ : total mass of air in the supply manifold, consisting of the sum of the instantaneous masses of oxygen, nitrogen and water vapor.

$x_{4}=m_{o_{2}, c a}$ : instantaneous oxygen mass in the stack's cathode. This state is affected by the oxygen consumed in the reaction, the amount of oxygen coming from the supply manifold and the oxygen mass outgoing through the return manifold.

$x_{5}=m_{N_{2}, \mathrm{ca}}$ : instantaneous mass of nitrogen inside the stack's cathode. It only relies on the incoming nitrogen from the supply manifold and the outgoing nitrogen that leaves the stack through the return manifold.

$x_{6}=p_{\mathrm{rm}}$ : total pressure inside the return manifold, consisting of the sum of the partial pressures of the gases that constitute the air.

The input $u(t)$ is the normalized voltage of the DC motor stator $V_{\mathrm{cm}}$. The normalization constant $\left(V_{\text {nor }}=180 \mathrm{~V}\right)$ is the maximum value of $V_{\mathrm{cm}}$ in the operating region. The normalization is performed in order to satisfy the hypothesis required in the design method developed in Section III-C. From the point of view of the SM control, this constraint guarantees $\left|u_{e q}\right|<1$.

\section{B. Control Objective}

The second stage in the control design procedure is to establish the control objective and, accordingly, define the sliding surface. In this case, the proposed objective is the optimization of the energy conversion of the FCGS, maximizing the net power generated by the system under different load conditions. This efficiency optimization can be achieved by regulating the oxygen mass flow entering to the stack cathode. If an adequate comburent flow is ensured through the stack, the load demand will be satisfied with minimum fuel consumption and, in addition, oxygen starvation will be averted.

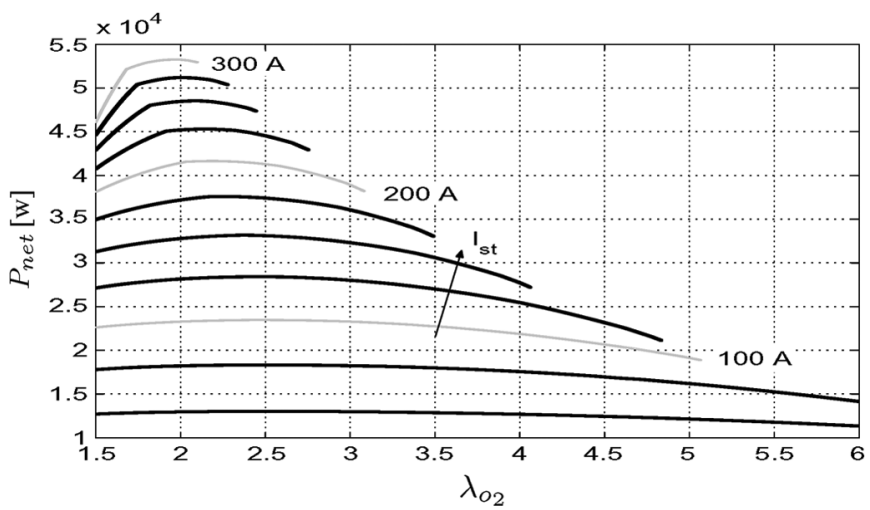

Fig. 2. Steady-state analysis of the system performance in different load conditions.

To accomplish such optimal comburent flow is equivalent to maintain the oxygen excess ratio of the cathode in an optimal value. The oxygen excess ratio or stoichiometry is defined as

$$
\lambda_{o_{2}}=\frac{W_{o_{2}, \text { in }}}{W_{o_{2}, \text { react }}}
$$

where $W_{o_{2}, \text { in }}$ is the oxygen partial flow in the cathode, which depends on the air flow released by the compressor $W_{\mathrm{cp}}$ and the vapor injected by the humidifier. $W_{o_{2} \text {,react }}$ is the oxygen flow consumed in the reaction, so it is directly related to the total stack current $\left(I_{\mathrm{st}}\right)$

$$
W_{o_{2}, \text { react }}=M_{O_{2}} \frac{n I_{\text {st }}}{4 F} .
$$

$M_{o 2}$ is the molar mass of oxygen, $n$ the total number of cells of the stack, and $F$ the Faraday constant.

The optimum value of $\lambda_{o_{2}}$ is determined from a thorough offline analysis of the open-loop system, considering changes in the current demand to the stack and a wide set of stoichiometry values. In Fig. 2, it can be observed that the optimum $\lambda_{o_{2}}$ depends on $I_{\mathrm{st}}$. However, in the FCGS under consideration it presents minor deviations all over the system operation range. Thus, a constant value of $\lambda_{o_{2}, \text { opt }}$ can be used. If this condition does not hold for other applications, then a variable $\lambda_{o_{2}, \text { opt }}$ can be easily obtained as a function of $I_{\mathrm{st}}$.

Note that other important advantage of the oxygenation control is the avoidance of oxygen starvation on the cathode side, given that it would only happen if $\lambda_{o_{2}}$ is allowed to go below 1 . Starvation is a significant problem that could lead to irreversible damages to the polymeric membranes if $\lambda_{o_{2}}<1$ persists for a long time.

Once $\lambda_{o_{2}, \text { opt }}$ is determined, the objective of keeping the oxygen excess ratio within optimal values can be written in terms of controlling the oxygen mass flow $\left(W_{o_{2}, \text { in }}\right)$. Then, the following mass flow reference can be obtained from (2) and (3):

$$
W_{o_{2}, \text { in }_{\text {ref }}}=\lambda_{o_{2}, \mathrm{opt}} M_{o_{2}} \frac{n I_{\mathrm{st}}}{4 F}
$$

where tracking $W_{o_{2}}$,in ref $_{\text {ref }}$ effectively implies $\lambda_{o_{2}}=\lambda_{o_{2}}$,opt .

Nevertheless, due to the fact that $W_{o_{2}}$,in is an internal unavailable variable of the FGCS, it is not practical to include it in the control algorithm. This problem can be circumvented by inferring information of $W_{O_{2}}$,in from an accessible variable of the 
system, such as the air mass flow delivered by the turbo compressor $W_{\text {cp. }}$. Under the aforementioned fixed humidification assumption, this variable is directly related to $W_{O_{2}}$, in through the supply manifold dynamics. Furthermore, once the manifold transient is extinguished, the relationship between $W_{\mathrm{cp}}$ and $W_{O_{2}, \text { in }}$ remains constant. Therefore, posing the control objective in terms of a tracking control problem of $W_{\mathrm{cp}}$, the operation of the stack close to its maximum efficiency points can be successfully achieved $\left(\lambda_{O_{2}}=\lambda_{o_{2}, \text { opt }}\right.$ is ensured for every load condition, once the supply manifold transient expires).

In the framework of the VSC theory, this control objective can be expressed as follows:

$$
s(x, t)=W_{\mathrm{cp}}-W_{\mathrm{air}_{\mathrm{ref}}}
$$

where $s$ is the sliding variable that must be steered to zero and $W_{\text {air }}$ ref is the air mass flow reference. The expression of the latter can be readily obtained from the air mass flow reference. Given that the molar fraction of oxygen in the air $\left(\chi_{o 2}\right)$ is known, the desired mass flow of dry air can be directly computed from (4) [3]

$$
W_{\text {dryair }_{\text {ref }}}=\frac{1}{\chi_{o 2}} W_{o_{2}, \mathrm{in}_{\mathrm{ref}}}=\frac{1}{\chi_{o 2}} \lambda_{o_{2}, \mathrm{opt}} M_{o_{2}} \frac{n I_{\mathrm{st}}}{4 F} .
$$

Then, taking into account the relative humidity of the air $\left(\Omega_{\mathrm{atm}}\right)$, the final expression of the air mass reference results

$$
W_{\text {air }_{\text {ref }}}=\left(1+\Omega_{\mathrm{atm}}\right) \frac{1}{\chi_{o 2}} \lambda_{o 2, \mathrm{opt}} M_{o 2} \frac{n I_{\mathrm{st}}}{4 F} .
$$

Note that for stable ambient conditions, the reference only depends on a single measurable variable, i.e., the stack current $I_{\mathrm{st}}$.

\section{Controller Design}

The sliding variable $s$ has relative degree one with respect to the control input $u$. Note that $s$ does not explicitly depend on the control input, while $u$ appears in the $\dot{s}$ expression (see Appendices II and III). In addition, the vector fields $f$ and $g$ are smooth enough in the operation range and information about $s$ is assumed to be available. Thus, differentiating the sliding variable twice, the following relations are derived:

$$
\begin{aligned}
\dot{s}= & \frac{\partial}{\partial t} s(t, x)+\frac{\partial}{\partial x} s(t, x) \cdot(f(x)+g(x, u)) \\
\ddot{s}= & \frac{\partial}{\partial t} \dot{s}(t, x, u)+\frac{\partial}{\partial x} \dot{s}(t, x, u) \cdot(f(x)+g(x, u)) \\
& +\frac{\partial}{\partial u} \dot{s}(t, x, u) \cdot \dot{u}(t) \\
= & \varphi(t, x, u)+\gamma(t, x, u) \dot{u}(t) .
\end{aligned}
$$

Due to readability reasons, the expressions of (8) and (9) are detailed in Appendix III.

Then, analyzing the system equations it is verified that the conditions required for SOSM control design are satisfied by the FC system (1). These are as follows.

1) The control values belong to the $\operatorname{closed} \operatorname{set} \mathcal{U}=\{u:|u| \leq$ $\left.U_{m}\right\}$, where $U_{m}$ is a real constant.

2) There exists $u_{1} \in(0,1)$ such that for any continuous function $u(t)$ with $|u(t)|>u_{1}$, there is $t_{1}$, such that $s(t) u(t)>$ 0 for each $t>t_{1}$. Hence, the control $u(t)=-\operatorname{sign}\left(s\left(t_{0}\right)\right)$,

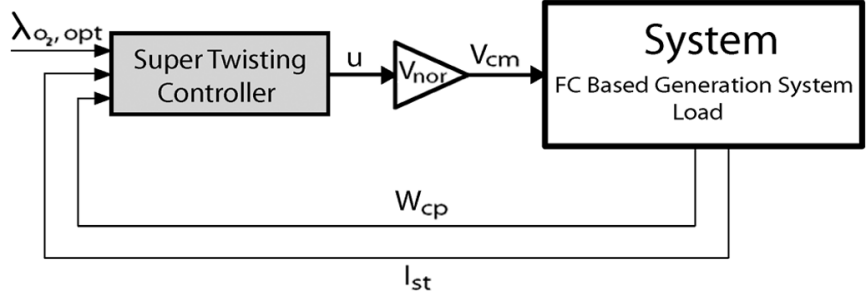

Fig. 3. Block diagram of the controlled system.

where $t_{0}$ is the initial value of time, provides hitting the surface $s=0$ in finite time. To this end, it has been defined in the design model $u=\left(V_{\mathrm{cm}} / V_{\text {nor }}\right)$ with $V_{\text {nor }}=180 \mathrm{~V}$.

3) Given $\dot{s}$, the total time derivate of the sliding variable $s$, there are positive constants $s_{0}, u_{0}<1, \Gamma_{m}, \Gamma_{M}$, such that if $|s|<s_{0}$ then

$0<\Gamma_{m} \leq \frac{\partial}{\partial u} \dot{s}(t, x, u) \leq \Gamma_{M} \quad \forall u \in \mathcal{U}, x \in \mathcal{X}$.

For the FCGS under consideration such bounds were obtained from a detailed analysis of the system structure together with comprehensive simulation studies, using a stabilizing control that maintains the system in a secure operation region. As a result, the following bounds were determined when considering $s_{0}=5 e^{-4}$ :

$$
\Gamma_{m}=0.5 \quad \Gamma_{M}=0.9 .
$$

4) In addition, a positive constant $\Phi=0.01$ has been computed, such that within the region $|s|<s_{0}$, the following inequality holds $\forall t, x \in \mathcal{X}, u \in \mathcal{U}$ :

$$
\left|\frac{\partial}{\partial t} \dot{s}(t, x, u)+\frac{\partial}{\partial x} \dot{s}(t, x, u) \cdot(f(x)+g(x, u))\right| \leq \Phi .
$$

Therefore, the stabilization problem of the system (1) with input-output dynamics (9) can be solved through the solutions of the following equivalent differential inclusion by applying SOSM:

$$
\ddot{s} \in[-\Phi, \Phi]+\left[\Gamma_{m}, \Gamma_{M}\right] \dot{u} .
$$

Using the information of the global bounds of $\varphi(\cdot)$ and $\gamma(\cdot)$, the final controller parameters can be designed. A schematic diagram of the developed control structure can be appreciated in Fig. 3.

In the sequel, it is developed a control law based on the supertwisting algorithm, which is one of the most popular algorithms among the SOSM algorithms [5], [6], [15]. The main advantage of this algorithm relies on its robustness to parametric uncertainties and disturbances when the conditions (10) and (11) hold. Besides, during online operation it only requires knowledge of the sign of the sliding variable. It is used for plants of relative degree one and the control $u$ can be given as a sum of two components

$$
\begin{aligned}
u(t) & =u_{1}(t)+u_{2}(t) \\
\dot{u}_{1}(t) & =-\gamma \operatorname{sign}(s) \\
u_{2}(t) & = \begin{cases}-\lambda\left|s_{0}\right|^{\rho} \operatorname{sign}(s), & \text { if }|s|>\left|s_{0}\right| \\
-\lambda|s|^{\rho} \operatorname{sign}(s), & \text { if }|s| \leq\left|s_{0}\right|\end{cases}
\end{aligned}
$$


TABLE I

VARIATIONS OF SYSTEM PARAMETERS

\begin{tabular}{|c|c|}
\hline Parameter & Variation \\
\hline Stack temperature $\left(T_{s t}\right)$ & $+10 \%$ \\
Cathode volume $\left(V_{c a}\right)$ & $+5 \%$ \\
Supply manifold volume $\left(V_{s m}\right)$ & $-10 \%$ \\
Atmospheric pressure $\left(p_{a t m}\right)$ & $+10 \%$ \\
Ambient temperature $\left(T_{a m b}\right)$ & $+10 \%$ \\
Return manifold volume $\left(V_{r m}\right)$ & $-10 \%$ \\
Motor constant $\left(K_{v}\right)$ & $-10 \%$ \\
Electric resistance of the motor $\left(R_{c m}\right)$ & $+5 \%$ \\
Compressor diameter $\left(d_{c}\right)$ & $+1 \%$ \\
Motor inertia $\left(J_{c p}\right)$ & $+10 \%$ \\
\hline
\end{tabular}

where $\gamma, \lambda$, and $\rho$ are design parameters. The corresponding sufficient conditions for the finite time convergence to the sliding manifold are [5]

$$
\begin{aligned}
\gamma & >\frac{\Phi}{\Gamma_{m}} \\
\lambda^{2} & \geq \frac{4 \Phi}{\Gamma_{m}^{2}} \frac{\Gamma_{M}(\gamma+\Phi)}{\Gamma_{m}(\gamma-\Phi)} \\
0 & <\rho \leq 0.5
\end{aligned}
$$

The controller parameters were designed in order to fulfill the previous conditions, so it is ensured that the control has sufficient authority to affect the sign of $\ddot{s}$. Then, the establishment of the SOSM in finite time is guaranteed. The solutions of the systems (1), (13), and inclusion (12) are understood in Fillipov sense.

To make the final tuning of the controller parameters it is recommended to consider not only the behavior of the oxygen stoichiometry, but also the electric quality of the net power output of the system. Therefore, given the direct effect of the control signal on the electric output, the super twisting controller parameters were selected in order to have a low content of high frequency components in the control. After an iterative refining procedure, the most adequate set of parameters resulted as follows:

$$
\gamma=2 \quad \lambda=2 \quad \rho=0.5
$$

It will be shown in Section IV that, under the influence of the control (13), the phase portrait of the system $(s-\dot{s}$ plane) presents the characteristic non-monotonous behavior of the super twisting algorithm. The trajectories converge to the origin in finite time, twisting around the center during the reaching mode.

\section{Simulation Results}

To verify the efficiency of the proposed controller (13) dealing with model uncertainties, external disturbances, and a wide range of current demand, a number of simulation studies were performed. To assess the controller performance under realistic operation conditions, simulation tests were conducted controlling the comprehensive ninth-order nonlinear model developed by Pukrushpan. The simulation model incorporates the original lookup tables representing parameters characteristics obtained from experimental data. Moreover, extra uncertainty

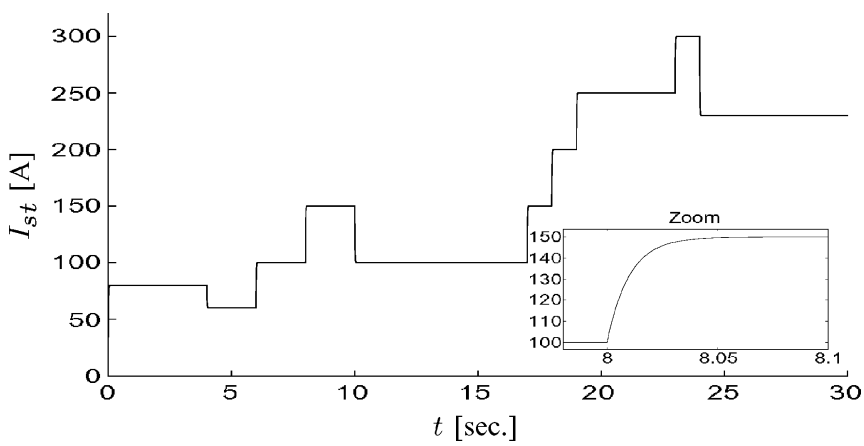

Fig. 4. Drained current from the stack.

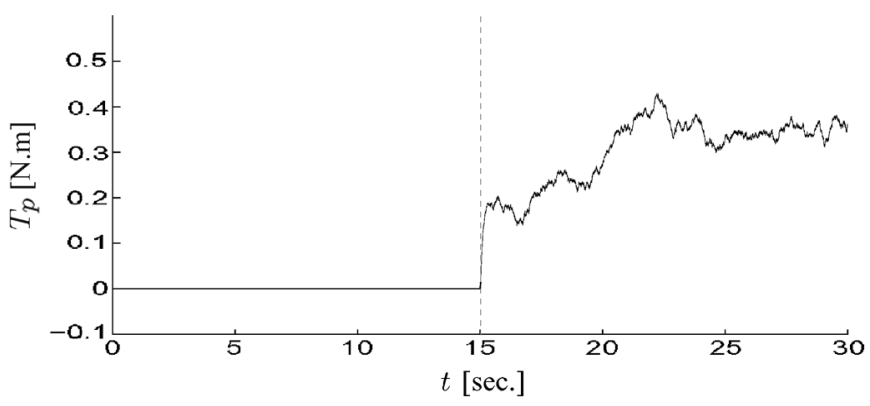

Fig. 5. Dynamic disturbance.

has been incorporated in several parameters of the system, verifying that conditions (10) and (11) hold (see Table I).

The features of the designed controller are examined through simulation tests, which aim to demonstrate its nominal performance and its robust tracking characteristics in the presence of unknown disturbances and uncertainties. The system nominal parameters of the design model are listed in Appendix I.

To this end, series of drained current (from 60 to $300 \mathrm{~A}$ ) were designed in order to illustrate the air regulation in a wide range of operation points. A sequence of abrupt current variations was generated from a filtered steps series (see Fig. 4). Besides, note that rather significative changes in the amplitude of the load demand were considered to test the proficiency of the controller under exacting conditions of operation.

In addition, an unknown torque disturbance modelled as a noisy quadratic function of the angular speed $\left(\omega_{\mathrm{cp}}\right)$ was included (see Fig. 5). This friction is set to start at $t=15 \mathrm{~s}$ and its expression can be written as follows:

$$
T_{p}=\omega_{\mathrm{cp}}(t)^{2} B_{1}+\omega_{\mathrm{cp}}(t) B_{2}+e(t)
$$

with $B_{1}=10^{-9}\left[\mathrm{~N} \cdot \mathrm{m} / \mathrm{s}^{2}\right], B_{2}=20.10^{-6}[\mathrm{~N} \cdot \mathrm{m} / \mathrm{s}]$ and $e(t)$ corresponds to a band limited noise component.

The optimum values of $\lambda_{o 2}$ previously referred, were determined performing offline simulations. In this way, the values $\lambda_{o 2, \text { opt }}$ that enable the system to work in its maximum net power point for each load condition were obtained (see Fig. 2). Due to the relatively small variation of $\lambda_{o 2 \text {,opt }}$ along the entire operation domain, it was adopted a unique optimum value to use as reference, $\lambda_{o 2, \mathrm{opt}}=2,05$. When this condition is reached, it can be assured that the system is working in the neighborhood of its maximum power generation point for different values of drained current. 


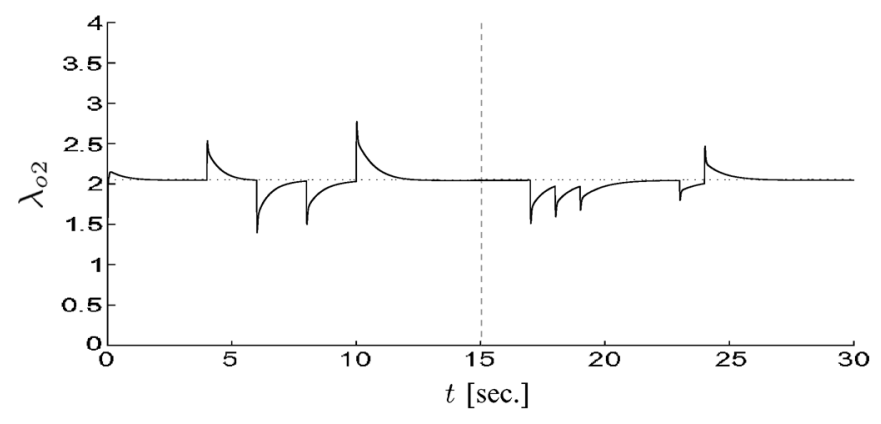

Fig. 6. Oxygen excess ratio.

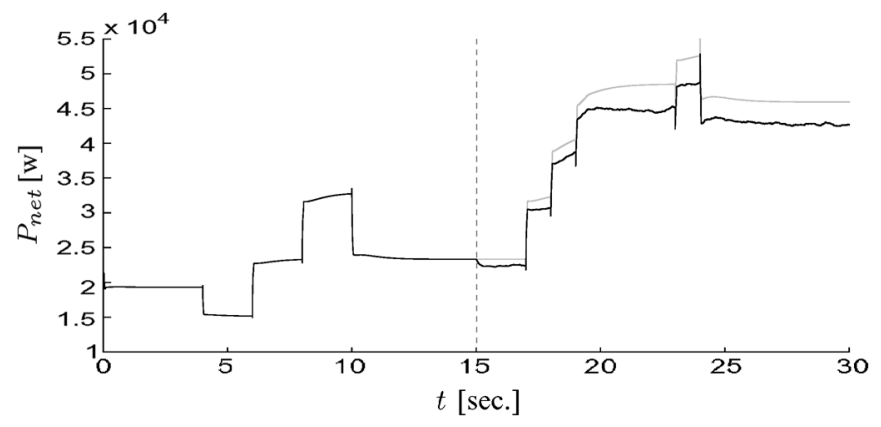

Fig. 7. Net power delivered by the system.

In Fig. 6 and the sequel, the black line depicts the system trajectory in presence of uncertainties and friction disturbances, while the gray line shows the behavior of the undisturbed system. Note that in Figs. 6, 8, and 9 both lines are superimposed.

The behavior of the oxygen stoichiometry $\left(\lambda_{o 2}\right)$ under different load conditions is depicted in Fig. 6. It can be observed that this variable satisfactorily follows the reference in spite of uncertainties and disturbances. Note that for the particular test under consideration, the transient due to some steps is not yet extinguished when the next one arises. The reason for this is that for better visualization of $\lambda_{o 2}$ time evolution, the whole series of steps had to be compacted into a brief lapse of $30 \mathrm{~s}$. This fact combined with the abrupt changes in $I_{s t}$ are responsible for the overlapping. However, even facing the excessively rigorous conditions of this simulation test, it is appreciated that the system response is not only robust but also rapid (it can be inferred from Fig. 6 that the system practically would reach the desired $\lambda_{o 2 \text {,opt }}$ in less than $3 \mathrm{~s}$ ).

Figs. 7 and 8 exhibit the dynamic behavior of the net power generated by the system $\left(P_{\text {net }}\right)$ and the stack voltage $\left(V_{\mathrm{st}}\right)$, the former displaying a desirable low content of high frequency components. Note that the noticeable reduction in the net power of the disturbed system (after $t=15 \mathrm{~s}$ ) is the expected consequence of the additional load incorporation, represented by the torque disturbance of Fig. 5.

Fig. 9 shows the temporal evolution of the sliding variable $(s(t))$. Once the reaching mode is over, the controller keeps the system operating on the sliding surface $(s(t)=0$, i.e. maintaining $\lambda_{o 2}$ in its optimum value), despite the coexistence of parameters uncertainties, external disturbances and important load variations. Note that during the reaching mode, $s(t)$ exhibits the typical overshooting behavior of a system controlled by a super twisting algorithm.

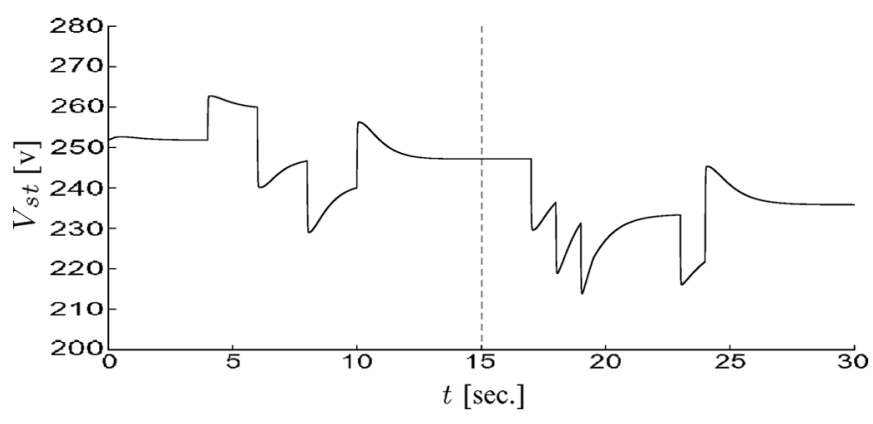

Fig. 8. Stack output voltage.

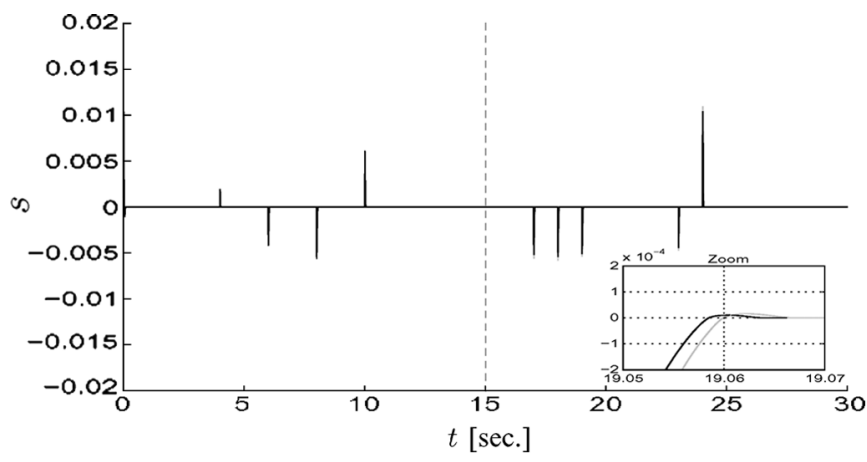

Fig. 9. Sliding variable.

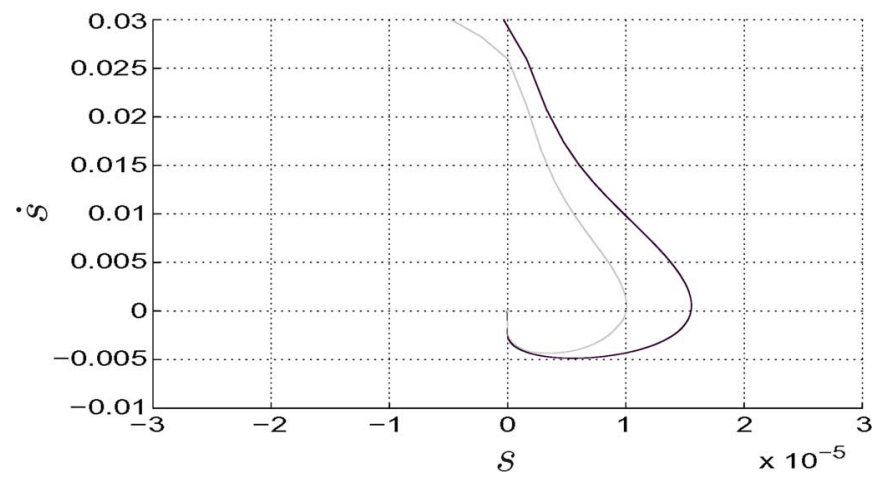

Fig. 10. $s(t)$ versus $\dot{s}(t)$.

The typical twisting effect imposed by the super twisting controller can be better appreciated in Fig. 10, where the phase portrait of the system is plotted for an arbitrary lapse between 19.2 and $19.3 \mathrm{~s}$.

Notice that the stability of the closed-loop system is guaranteed because the differential inclusion (12) is satisfied.

Finally, in Fig. 11, the time evolution of the control signal is presented $(u(t))$. Note the increase in the control effort after the friction disturbance appears.

\section{CONCLUSION}

A control system that globally solves the breathing problem of a fuel cell based generation system was developed. The plant configuration is based on a 75-kW PEM stack, fed by high pressure hydrogen and air delivered by a $14-\mathrm{kW}$ turbo compressor. A second-order sliding mode strategy was used to solve the control problem of power optimization and oxygen starvation. The main advantages of the adopted nonlinear control design approach applied to the FC stack are as follows: 


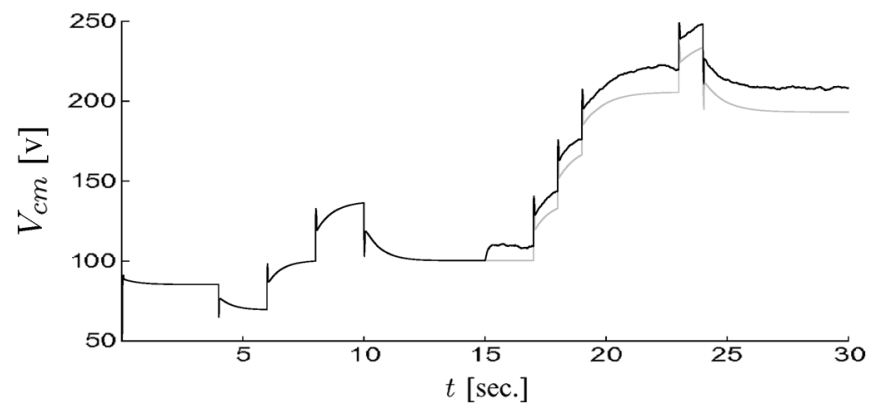

Fig. 11. Control signal.

- robustness to parameter uncertainties and external disturbances;

- guaranteed extended range of operation, in spite of the highly nonlinear nature of plant;

- control law only depends on two measurable variables, namely the stack current and the compressor air flow, therefore no observer or state estimation is required;

- structure of the algorithm is simple, thus low online computational burden is required.

The proposed control strategy is based on a super twisting algorithm that robustly solves the stabilization problem avoiding chattering effects. The control development is based on a design model of the plant especially rebuilt for SOSM purposes. The analysis and final validation of the designed controller were performed through comprehensive simulations using the complete Pukrushpan's model, considering several load conditions, uncertainties in model parameters and disturbances. The simulation tests were highly satisfactory, showing the suitability of the SOSM algorithm to control PEM-type autonomous FC systems. Due to the encouraging results of the proposed strategy applied to this plant, it is planned to continue future works in the same research line aiming to improve and compare new control strategies based on SOSM algorithms.

\section{APPENDIX I \\ PARAMETERS}

\section{Differential Equations Parameters}

$B_{1}=n_{\mathrm{cm}} /\left(R_{\mathrm{cm}} J_{\mathrm{cp}}\right) ; B_{2}=K_{v} n_{\mathrm{cm}} /\left(R_{\mathrm{cm}} J_{\mathrm{cp}}\right) ;$ $B_{3}=-C_{p} T_{\mathrm{atm}} \phi_{\max } \rho_{a} \pi d_{c}^{2} K_{U c} \delta /\left(4 n_{\mathrm{cp}} \theta^{1 / 2} e f_{\mathrm{mec}} J_{\mathrm{cp}}\right)$; $B_{4}=(\gamma-1) / \gamma ; B_{5}=2 C_{p} T_{\mathrm{cp}, \mathrm{in}} K_{U c}^{-2} ;$ $B_{6}=\phi_{\max } \rho_{a} \pi d_{c}^{2} K_{U c} \delta T_{\mathrm{atm}} \gamma R_{a} /\left(4 \theta^{1 / 2} V_{\mathrm{sm}}\right)$; $B_{7}=K_{\text {sm,out }} \gamma ; B_{8}=B_{7} p_{v, \text { ca }} ; B_{9}=B_{7} R_{N_{2}} T_{\text {st }} / V_{\text {ca }}$; $B_{10}=B_{7} R_{O_{2}} T_{\text {st }} / V_{\text {ca }} ; B_{11}=\phi_{\max } \rho_{a} \pi d_{c}^{2} K_{U c} \delta /\left(4 \theta^{1 / 2}\right)$; $B_{12}=K_{\mathrm{sm}, \text { out }} ; B_{13}=K_{\mathrm{sm}, \text { out }} p_{v, \mathrm{ca}} ; B_{14}=$ $K_{\mathrm{sm}, \text { out }} R_{N_{2}} T_{\mathrm{st}} / V_{\mathrm{ca}} ; \quad B_{15}=K_{\mathrm{sm}, \text { out }} R_{\mathrm{O}_{2}} T_{\mathrm{st}} / V_{\text {ca }}$; $B_{16}=M_{v} \phi_{\mathrm{des}} p_{\mathrm{sat}, T_{\mathrm{cl}}} K_{\mathrm{sm}, \mathrm{out}} / M_{a} ; B_{17}=B_{16} p_{v, \mathrm{ca}} ;$ $B_{18}=B_{16} R_{N_{2}} T_{\text {st }} / V_{\text {ca }} ; B_{19}=B_{16} R_{O_{2}} T_{\text {st }} / V_{\text {ca }} ; B_{20}=$ $M_{v} \phi_{\mathrm{atm}} p_{\mathrm{sat}, T_{\mathrm{atm}}} /\left(M_{a} p_{\mathrm{atm}}\right) ; B_{21}=\phi_{\mathrm{atm}} p_{\mathrm{sat}, T_{\mathrm{atm}}} / p_{\mathrm{atm}} ;$ $B_{22}=M_{v} \phi_{\text {ca,in }} p_{\text {sat }, T_{\mathrm{cl}}}\left(Y_{O_{2}, \text { ca, in }} M_{O_{2}}+(1-\right.$ $\left.\left.Y_{\mathrm{O}_{2}, \mathrm{ca}, \mathrm{in}}\right) M_{N_{2}}\right)^{-1} ; B_{23}=\phi_{\mathrm{des}} p_{\mathrm{sat}, T_{\mathrm{cl}}}-\phi_{\mathrm{ca}, \text { in }} p_{\mathrm{sat}, T_{\mathrm{cl}}}$; $B_{24}=R_{O_{2}} T_{\mathrm{st}} M_{\mathrm{O}_{2}} k_{\text {ca, out }} / V_{\text {ca }} ; B_{25}=B_{24} p_{v, \text { ca }}$; $B_{26}=B_{24} R_{N_{2}} T_{\text {st }} / V_{\text {ca }} ; B_{27}=B_{24} R_{O_{2}} T_{\text {st }} / V_{\text {ca }}$; $B_{28}=R_{N_{2}} T_{\text {st }} / V_{\text {ca }} ; B_{29}=R_{O_{2}} T_{\text {st }} / V_{\text {ca }} ; B_{30}=B_{24} / k_{\text {ca }, \text { out }}$; $B_{31}=R_{O_{2}} T_{\text {st }} M_{N_{2}} / V_{\text {ca }} ; B_{32}=M_{O_{2}} n /(F 4) ; B_{33}=$
$1-Y_{O_{2}, \text { ca,in }} M_{O_{2}} /\left(Y_{O_{2}, \text { ca,in }} M_{O_{2}}+\left(1-Y_{O_{2}, \text { ca,in }}\right) M_{N_{2}}\right)$; $B_{34}=k_{\text {ca }, \text { out }} ; B_{35}=k_{\text {ca }, \text { out }} p_{v, \text { ca }} ; B_{36}=k_{\mathrm{ca}, \text { out }} R_{N_{2}} T_{\mathrm{st}} / V_{\mathrm{ca}}$; $B_{37}=k_{\text {ca }, \text { out }} R_{O_{2}} T_{\mathrm{st}} / V_{\mathrm{ca}} ; B_{38}=R_{a} T_{\mathrm{st}} / V_{\mathrm{rm}}$; $B_{39}=B_{38} p_{a 1} / \operatorname{std}_{a}^{5} ; \quad B_{40}=B_{38} p_{a 2} / \mathrm{std}_{a}^{4}$; $B_{41}=B_{38} p_{a 3} / \operatorname{std}_{a}^{3} ; B_{42}=B_{38} p_{a 4} / \mathrm{std}_{a}^{2} ;$ $B_{43}=B_{38} p_{a 5} / \operatorname{std}_{a} ; B_{44}=B_{38} p_{a 6} ; B_{45}=\operatorname{mean}_{a} ;$ $B_{46}=B_{34} B_{38} ; B_{47}=B_{35} B_{38} ; B_{48}=B_{36} B_{38}$; $B_{49}=B_{37} B_{38} ; B_{50}=B_{33} B_{16} ; B_{51}=B_{50} p_{v, \text { ca }}$; $B_{52}=B_{50} B_{28} ; B_{53}=B_{50} B_{29} ; B_{54}=B_{33} B_{12}$; $B_{55}=B_{33} B_{13} ; B_{56}=B_{33} B_{14} ; B_{57}=B_{33} B_{15}$; $B_{58}=M_{v} p_{v, \mathrm{ca}} ; B_{59}=X_{O_{2}, \mathrm{ca}, \mathrm{in}} B_{16} ; B_{60}=X_{O_{2}, \mathrm{ca}, \mathrm{in}} B_{17}$; $B_{61}=X_{O_{2}, \mathrm{ca}, \mathrm{in}} B_{18} ; B_{62}=X_{O_{2}, \mathrm{ca}, \mathrm{in}} B_{19}$; $B_{63}=X_{O_{2}, \mathrm{ca}, \mathrm{in} B_{12}} ; B_{64}=X_{\mathrm{O}_{2}, \mathrm{ca}, \mathrm{in} B_{13}}$; $B_{65}=X_{O_{2}, \text { ca }, \text { in }} B_{14} ; B_{66}=X_{O_{2}, \text { ca }, \text { in }} B_{15} ; B_{67}=B_{30}-B_{31}$; $B_{68}=B_{29} M_{O_{2}}-B_{31} ; B_{69}=\beta B_{5} \Phi_{\max }^{-1} ; K_{U c}=d_{c} /(2 \sqrt{\theta})$.

\section{Physical Parameters (Metric System Units)}

$\beta=14 ; \gamma=1.4 ; \delta=p_{\mathrm{cp}, \mathrm{in}} / 1 ; \theta=T_{\mathrm{cp}, \mathrm{in}} / 298 ; \rho_{a}=$ $1.23 ; \phi_{\mathrm{atm}}=0.5 ; \phi_{\mathrm{ca}, \mathrm{in}}=1 ; \phi_{\mathrm{des}}=1 ; \phi_{\max }=1.55 \times$ $10^{-3} ; \Phi_{\max }=0.197 ; d_{c}=0.2286 ; e f_{\mathrm{mec}}=0.9 ; k_{\mathrm{ca}, \text { out }}=$ $2.17 \times 10^{-6} ; m_{v, \text { ca }, \max }=0.0028 ; \operatorname{mean}_{a}=2.5 \times 10^{5} ; n=$ $381 ; n_{\mathrm{cm}}=1 ; n_{\mathrm{cp}}=0.775 ; p_{a_{1}}=0.0012 ; p_{a_{2}}=-0.0019$; $p_{a_{3}}=-0.0015 ; p_{a_{4}}=0.0021 ; p_{a_{5}}=0.027 ; p_{a_{6}}=0.078$ $p_{\mathrm{amb}}=1 ; p_{\mathrm{atm}}=101325 ; p_{\mathrm{cp}, \mathrm{in}}=p_{\mathrm{amb}} ; p_{\mathrm{sat}, \mathrm{Tatm}}=3.14 \times$ $10^{3} ; p_{\mathrm{sat}, \mathrm{Tcl}}=47.06 \times 10^{3} ; p_{v, \mathrm{ca}}=m_{v, \mathrm{ca}, \max } R_{v} T_{\mathrm{st}} / V_{\mathrm{ca}}$; $\operatorname{std}_{a}=8.66 \times 10^{4} ; C_{p}=1004 ; F=96485 ; J_{\mathrm{cp}}=5 \times 10^{-5}$; $K_{\mathrm{sm}, \text { out }}=0.36 \times 10^{-5} ; K_{v}=0.0153 ; M_{a}=28.84 \times 10^{-3}$; $M_{N_{2}}=28 \times 10^{-3} ; M_{O_{2}}=32 \times 10^{-3} ; M_{v}=18.02 \times$ $10^{-3} ; R_{a}=2.869 \times 10^{2} ; R_{\mathrm{cm}}=1.2 ; R_{O_{2}}=259.8 ; R_{N_{2}}=$ $296.8 ; R_{v}=461.5 ; T_{\mathrm{amb}}=298 ; T_{\mathrm{atm}}=T_{\mathrm{amb}} ; T_{\mathrm{cp}, \mathrm{in}}=$ $T_{\mathrm{amb}} ; T_{\mathrm{st}}=353 ; T_{\mathrm{fc}}=T_{s t} ; V_{\mathrm{ca}}=0.01 ; V_{\mathrm{rm}}=0.005 ;$ $V_{\mathrm{sm}}=0.02 ; X_{\mathrm{o} 2, \mathrm{ca}, \text { in }}=\left(Y_{\mathrm{o} 2, \mathrm{ca}, \text { in }} M_{\mathrm{O}_{2}}\right) /\left(Y_{\mathrm{O}_{2}, \mathrm{ca}, \text { in }} M_{\mathrm{O}_{2}}+(1-\right.$ $\left.\left.Y_{O_{2}, \text { ca,in }}\right) M_{N_{2}}\right) ; Y_{o 2, \text { ca,in }}=0.21$.

\section{APPENDIX II}

State Space Equations

\section{MODEL EQUATIONS}

$$
\begin{aligned}
\dot{x}_{1}= & B_{3} n(x)(1-d(x))+\left(B_{1} u^{2} x_{1}^{-1}-B_{2} u\right) \\
\dot{x}_{2}= & B_{6}(1-d(x)) x_{1}\left(1+n(x) n_{\mathrm{cp}}^{-1}\right) \\
& -\left(x_{2}^{2} B_{7}-x_{2} B_{8}-x_{2} x_{5} B_{9}-x_{2} x_{4} B_{10}\right) x_{3}^{-1} \\
\dot{x}_{3}= & B_{11}(1-d(x)) x_{1}-x_{2} B_{12}+B_{13}+x_{5} B_{14}+x_{4} B_{15} \\
\dot{x}_{4}= & \left(\left(x_{2} B_{59}-B_{60}-x_{5} B_{61}-x_{4} B_{62}\right)\left(x_{2}-x_{2} B_{21}\right)^{-1} e(x)\right. \\
& \left.+\left(x_{2} B_{63}-B_{64}-x_{5} B_{65}-x_{4} B_{66}\right) e(x)\right) k(x) \\
& -x_{4}\left(B_{25}+x_{5} B_{26}+x_{4} B_{27}-x_{6} B_{24}\right) j(x) x_{4}^{-1} \\
& \times\left(j(x) B_{67}+M_{N 2}\right)^{-1} m(x)-I s t B_{32} \\
\dot{x}_{5}= & \left(\left(x_{2} B_{50}-B_{51}-x_{5} B_{52}-x_{4} B_{53}\right)\left(x_{2}-x_{2} B_{21}\right)^{-1} e(x)\right. \\
& \left.+\left(x_{2} B_{54}-B_{55}-x_{5} B_{56}-x_{4} B_{57}\right) e(x)\right) k(x) \\
& -\left(1-j(x) B_{30}\left(j(x) B_{68}+M_{N 2}\right)^{-1}\right) \\
& \times\left(B_{35}+x_{5} B_{36}+x_{4} B_{37}-x_{6} B_{34}\right) m(x) \\
\dot{x}_{6}= & B_{47}+x_{5} B_{48}+x_{4} B_{49}-x_{6} B_{46}-B_{39} c(x)^{5} \\
& -B_{40} c(x)^{4}-B_{41} c(x)^{3}-B_{42} c(x)^{2}-B_{43} c(x)-B_{44} .
\end{aligned}
$$

Output

$$
y=W_{\mathrm{cp}}=B_{11}(1-d(x)) x_{1}
$$


where

$$
\begin{aligned}
d(x) & =e^{B_{69}\left(\left(\frac{x_{2}}{p_{\text {atm }}}\right)^{B_{4}}-1\right) x_{1}^{-2}-\beta} \\
e(x) & =\left(1+\frac{x_{2} B_{20}}{x_{2}-x_{2} B_{21}}\right)^{-1} ; c(x)=x_{6}-B_{45} \\
j(x) & =\frac{x_{4}}{x_{5} B_{28}+x_{4} B_{29}} \\
k(x) & =\left(1+\frac{B_{22}}{x_{2}-x_{2} B_{21}+B_{23}}\right)^{-1} ; \\
m(x) & =\left(1+B_{58}\left(j(x) B_{68}+M_{N 2}\right)^{-1} j(x) x_{4}^{-1}\right)^{-1} ; \\
n(x) & =\left(\frac{x_{2}}{p_{\text {atm }}}\right)^{B_{4}}-1 .
\end{aligned}
$$

\section{APPENDIX III}

SLIDING VARIABLE AND ITS TIME DERIVATIVES

$$
\begin{aligned}
s(x, t)= & W_{\mathrm{cp}}-W_{\mathrm{air}_{\mathrm{ref}}}=B_{11}(1-d(x)) x_{1} \\
& -\left(1+\Omega_{\mathrm{atm}}\right) \frac{1}{\chi_{\mathrm{o} 2}} \lambda_{\mathrm{o} 2, \mathrm{opt}} M_{o 2} \frac{n I_{\mathrm{st}}}{4 F} \\
\dot{s}(t)= & B_{11}\left(\dot{x}_{1} a(x)+\dot{x}_{2} b(x)\right)-2.8 \times 10^{-4} \frac{d I_{\mathrm{st}}}{d t}
\end{aligned}
$$

where

$$
\begin{aligned}
a(x) & =a_{1}(x)+a_{21}(x) a_{22}(x) x_{1} ; a_{1}(x)=1-d(x) ; \\
a_{21}(x)= & -d(x) ; a_{22}(x)=-2 B_{69} n(x) x_{1}^{-3} \\
b(x)= & b_{1}(x) b_{2}(x) x_{1} ; \\
b_{1}(x)= & a_{21}(x) ; b_{2}(x)=\frac{B_{69} B_{4} x_{2}^{B_{4}-1}}{x_{1}^{2} p_{\text {atm }}^{B_{4}}} \\
\ddot{s}(t)= & B_{11}\left(\ddot{x}_{1} a(x)+\dot{x}_{1} \dot{a}(x)+\ddot{x}_{2} b(x)+\dot{x}_{2} \dot{b}(x)\right) \\
& \quad-2.8 \times 10^{-4} \frac{d^{2} I_{s t}}{d t^{2}}=\varphi(t, x)+\gamma(t, x) \dot{u}(t)
\end{aligned}
$$

where

$$
\begin{aligned}
\varphi(t, x)= & {\left[\frac{\partial \dot{s}}{\partial x_{1}} \frac{\partial \dot{s}}{\partial x_{2}} \frac{\partial \dot{s}}{\partial x_{3}} \frac{\partial \dot{s}}{\partial x_{4}} \frac{\partial \dot{s}}{\partial x_{5}} \frac{\partial \dot{s}}{\partial x_{6}}\right] } \\
& \times(f(x)+g(x, u)) \\
\gamma(t, x)= & \frac{\partial}{\partial u} \dot{s}(x, u) \\
= & \left(2 B_{11} B_{69} n(x) d(x) x_{1}^{-2} \psi_{\max }^{-1}+B_{11}(1-d(x))\right) \\
& \times\left(2 u B_{1} x_{1}^{-1}-B_{2}\right) .
\end{aligned}
$$

\section{ACKNOWLEDGMENT}

The authors would like to thank Dr. J. Pukrushpan and Prof. A. Stefanopoulou for the comprehensive dynamic model of the fuel cell system and related material they provided us to develop our control system.

\section{REFERENCES}

[1] J. Larminie and A. Dicks, Fuel Cell Systems Explained, 2nd ed. New York: Wiley, 2003.

[2] C. Kunusch, "Second order sliding mode control of a fuel cell stack using a twisting algorithm," M.S. thesis, Electr. Dept., Nat. Univ. La Plata, Argentina, Mar. 2006.

[3] J. Pukrushpan, A. Stefanopoulou, and H. Peng, "Control of fuel cell breathing," IEEE Control Syst. Mag., vol. 24, no. 2, pp. 30-46, Apr. 2004.

[4] G. Bartolini, A. Ferrara, and E. Usai, "Second order VSC for non linear systems subjected to a wide class of uncertainty conditions," in Proc. IEEE Workshop Variable Structure Syst., 1996, pp. 49-54.

[5] A. Levant, "Sliding order and sliding accuracy in sliding mode control," Int. J. Control, vol. 58, no. 6, pp. 1247-1263, 1993.

[6] L. Fridman and A. Levant, "Higher Order Sliding Modes," in Sliding Mode Control in Engineering. New York: Marcel Dekker, Inc., 2002, ch. 3, pp. 53-101.

[7] L. Fridman and A. Levant, Robust Control Variable Structure and Lyapunov Techniques. London, U.K.: Springer Verlag, 1996, ch. 1, pp. 106-133, no. 217.

[8] J. Pukrushpan, A. Stefanopoulou, and H. Peng, Control of Fuel Cell Power Systems. London, U.K.: Springer, 2004.

[9] A. Sabanovic, L. M. Fridman, and S. Spurgeon, Variable Structure Systems: From Principles to Implementation. London, U.K.: IEE, 2004.

[10] S. Spurgeon and R. Davies, "A nonlinear control strategy for robust sliding mode performance in the presence of unmatched uncertainty," Int. J. Control, vol. 57, no. 5, pp. 1107-1123, 1993.

[11] H. Sira-Ramirez, "Differential geometric methods in variable structure control," Int. J. Control, vol. 48, no. 5, pp. 1359-1390, 1988.

[12] V. Utkin, J. Gulder, and J. Shi, Sliding Mode Control in Electro-Mechanical Systems. London, U.K.: Taylor and Francis, 1999.

[13] A. Levant, "Higher order sliding: Differentiation and black-box control," in Proc. IEEE Conf. Dec. Control, Dec. 2000, vol. 2, pp. 1703-1708.

[14] G. Bartolini, A. Ferrara, and E. Usai, "Chattering avoidance by second order sliding mode control," IEEE Trans. Autom. Control, vol. 43, no. 2, pp. 241-246, Feb. 1998.

[15] I. Boiko and L. Fridman, "Analysis of chattering in continuous slidingmode controllers," IEEE Trans. Autom. Control, vol. 50, no. 9, pp. 1442-1446, Sep. 2005. 